\title{
Solution and Estimation of RE Macromodels with Optimal Policy
}

\author{
Paul Söderlind* \\ Stockholm School of Economics
}

December 30, 1998

\begin{abstract}
Macro models of monetary policy typically involve forward looking behavior. Except in rare circumstances, we have to apply some numerical method to find the the optimal policy and the rational expectations equilibrium. This paper summarizes a few useful methods, and shows how they can be combined with a Kalman filter to estimate the deep model parameters with maximum likelihood. Simulations of a macro model with staggered price setting, interest rate elastic output, and optimal monetary policy illustrate the properties of this estimation approach.

Keywords: Unstable roots, Schur decomposition, Kalman filter estimation

JEL Classification Numbers: C32, C61, E52
\end{abstract}

\section{Optimal Policy in Linear Rational Expectations Models}

\subsection{A Class of Models}

The economies discussed in this paper evolve according to

$$
\left[\begin{array}{l}
x_{1 t+1} \\
\mathrm{E}_{t} x_{2 t+1}
\end{array}\right]=A\left[\begin{array}{l}
x_{1 t} \\
x_{2 t}
\end{array}\right]+B u_{t}+\left[\begin{array}{l}
\varepsilon_{t+1} \\
\mathbf{0}_{n_{2} \times 1}
\end{array}\right],
$$

* Stockholm School of Economics and CEPR. Address: Stockholm School of Economics, PO Box 6501, SE-113 83 Stockholm, Sweden. E-mail: nepso@ hhs.se. 
where $x_{1 t}$ is an $n_{1} \times 1$ vector of predetermined variables (backward looking) with $x_{10}$ given, $x_{2 t}$ an $n_{2} \times 1$ vector of nonpredetermined (forward looking) variables, $u_{t}$ a $k \times 1$ vector of policy instruments, and $\varepsilon_{t+1}$ an $n_{1} \times 1$ vector of innovations to $x_{1 t}$ with covariance matrix $\Sigma$. Some of the elements in $x_{1 t}$ can be exogenous variables like a serially correlated productivity level. For notational convenience, define the vector $x_{t}=\left(x_{1 t}, x_{2 t}\right)$, which is $n \times 1$, where $n=n_{1}+n_{2}$. The matrices $A$ and $B$, and the properties of the shocks $\varepsilon_{t+1}$ are assumed to be constant functions of some structural (deep) model parameters.

The policy maker has the loss function

$$
J_{0}=\mathrm{E}_{0} \sum_{t=0}^{\infty} \beta^{t}\left(x_{t}^{\prime} Q x_{t}+2 x_{t}^{\prime} U u_{t}+u_{t}^{\prime} R u_{t}\right) .
$$

The matrices $Q$ and $R$ are (without loss of generality) symmetric. They, as well as the matrix $U$ and the discount factor $\beta$ are also constant functions the structural model parameters.

\subsection{Optimal Policy under Commitment}

The policy maker is here assumed to be able to commit to a constant policy rule. Form the Lagrangian from (2) and (1)

$L_{0}=\mathrm{E}_{0} \sum_{t=0}^{\infty} \beta^{t}\left[x_{t}^{\prime} Q x_{t}+2 x_{t}^{\prime} U u_{t}+u_{t}^{\prime} R u_{t}+2 \rho_{t+1}^{\prime}\left(A x_{t}+B u_{t}+\xi_{t+1}-x_{t+1}\right)\right]$,

where $\xi_{t+1}=\left(\varepsilon_{t+1}, x_{2 t+1}-\mathrm{E} x_{2 t+1}\right)$, and $x_{10}$ is given. The first order conditions with respect to $\rho_{t+1}, x_{t}$, and $u_{t}$ are

$\left[\begin{array}{lll}I_{n} & \mathbf{0}_{n \times k} & \mathbf{0}_{n \times n} \\ \mathbf{0}_{n \times n} & \mathbf{0}_{n \times k} & \beta A^{\prime} \\ \mathbf{0}_{k \times n} & \mathbf{0}_{k \times k} & -B^{\prime}\end{array}\right]\left[\begin{array}{l}x_{t+1} \\ u_{t+1} \\ \mathrm{E}_{t} \rho_{t+1}\end{array}\right]=\left[\begin{array}{lll}A & B & \mathbf{0}_{n \times n} \\ -\beta Q & -\beta U & I_{n} \\ U^{\prime} & R & \mathbf{0}_{k \times n}\end{array}\right]\left[\begin{array}{l}x_{t} \\ u_{t} \\ \rho_{t}\end{array}\right]+\left[\begin{array}{l}\xi_{t+1} \\ \mathbf{0}_{n \times 1} \\ \mathbf{0}_{k \times 1}\end{array}\right]$. 
Take expectations of (4) conditional on the information set in $t$. Then, expand $x_{t}$ and $\rho_{t}$ as $\left(x_{1 t}, x_{2 t}\right)$ and $\left(\rho_{1 t}, \rho_{2 t}\right)$ respectively, and reorder the rows by placing $\rho_{2 t}$ after $x_{1 t}$. Write the result as

$$
G \mathrm{E}_{t}\left[\begin{array}{l}
k_{t+1} \\
\lambda_{t+1}
\end{array}\right]=D\left[\begin{array}{l}
k_{t} \\
\lambda_{t}
\end{array}\right] \text {, where } k_{t}=\left[\begin{array}{l}
x_{1 t} \\
\rho_{2 t}
\end{array}\right] \text { and } \lambda_{t}=\left[\begin{array}{l}
x_{2 t} \\
u_{t} \\
\rho_{1 t}
\end{array}\right]
$$

The reason for this ordering is that we want to exploit the initial conditions for the vector $k_{t}$. First, the initial state vector, $x_{10}$, is given. Second, forward looking variables can be chosen freely in the initial period so their shadow prices, $\rho_{20}$, are zero (see (Currie and Levine 1993)).

The matrix $G$ in (5) is singular. (Sims 2001) and (Klein 2000) show how the generalized Schur decomposition can be used in this situation. In contrast to the algorithms in (Levine and Currie 1987) and (Backus and Driffill 1986), this approach allows a singular $R$ matrix and available software libraries give very quick solutions. The latter is particularly important in estimations, where the equilibrium has to be calculated for every iteration on the vector of structural parameters.

The following is an application of Klein's method. Given the square matrices $G$ and $D$, the decomposition gives the square complex matrices $Q, S, T$, and $Z$ such that

$$
G=Q S Z^{H} \text { and } D=Q T Z^{H}
$$

where $Z^{H}$ denotes the transpose of the complex conjugate of $Z !^{1} Q$ and $Z$ are unitary $\left(Q^{H} Q=Z^{H} Z=I\right)$, and $S$ and $T$ are upper triangular (see (Golub and van Loan 1989)). The decomposition can be reordered so the block corresponding to the stable generalized eigenvalues (the $i^{\text {th }}$ diagonal element of $T$ divided by the corresponding element in $S$ ) comes first $\left.\right|^{2}$ In most cases, stability requires a

\footnotetext{
${ }^{1}$ Not the same $Q$ as in 2); I am "recycling" notation here.

2 This decomposition is used to solve the generalized eigenvalue problem $D x=\lambda G x$.
} 
modulus of the generalized eigenvalue less than one. With unit root exogenous variables, the cutoff point could often be chosen slightly above one, provided the unstable eigenvalues of the endogenous part are sufficiently above unity.

Define the auxiliary variables

$$
\left[\begin{array}{l}
\theta_{t} \\
\delta_{t}
\end{array}\right]=Z^{H}\left[\begin{array}{l}
k_{t} \\
\lambda_{t}
\end{array}\right]
$$

Premultiply (5) with the non-singular matrix $Q^{H}$, use (7), and partition $S$ and $T$ conformably with $\theta_{t}$ and $\delta_{t}$

$$
\left[\begin{array}{ll}
S_{\theta \theta} & S_{\theta \delta} \\
\mathbf{0} & S_{\delta \delta}
\end{array}\right] \mathrm{E}_{t}\left[\begin{array}{c}
\theta_{t+1} \\
\delta_{t+1}
\end{array}\right]=\left[\begin{array}{ll}
T_{\theta \theta} & T_{\theta \delta} \\
\mathbf{0} & T_{\delta \delta}
\end{array}\right]\left[\begin{array}{l}
\theta_{t} \\
\delta_{t}
\end{array}\right]
$$

Since the lower right block contains the unstable roots, it must be the case that $\delta_{t}=\mathbf{0}$ for all $t$ in order to get a stable solution. The remaining equations are then $S_{\theta \theta} \mathrm{E}_{t} \theta_{t+1}=T_{\theta \theta} \theta_{t}$, which we solve as

$$
\mathrm{E}_{t} \theta_{t+1}=S_{\theta \theta}^{-1} T_{\theta \theta} \theta_{t}
$$

since $S_{\theta \theta}$ is invertible (the determinant of the triangular $S_{\theta \theta}$ equals the product of the diagonal elements, which are all non-zero as a consequence of how the matrices were reordered).

Premultiply (7) with $Z$ and partition conformably with $k_{t}, \lambda_{t}, \theta_{t}$ and $\delta_{t}$,

$$
\begin{aligned}
{\left[\begin{array}{c}
k_{t} \\
\lambda_{t}
\end{array}\right] } & =\left[\begin{array}{ll}
Z_{k \theta} & Z_{k \delta} \\
Z_{\lambda \theta} & Z_{\lambda \delta}
\end{array}\right]\left[\begin{array}{l}
\theta_{t} \\
\delta_{t}
\end{array}\right] \\
& =\left[\begin{array}{l}
Z_{k \theta} \\
Z_{\lambda \theta}
\end{array}\right] \theta_{t},
\end{aligned}
$$

since $\delta_{t}=\mathbf{0}$. We know that $k_{0}=\left(x_{10}, \mathbf{0}_{n_{2} \times 1}\right)$, so we can solve for $\theta_{0}$ from (10) if $Z_{k \theta}$ is invertible. A necessary condition is that (5) has the "saddle path"

The results in the text hold also for the real generalized Schur decomposition where $Q$ and $Z$ are real orthogonal matrices, $S$ is real and upper triangular, and $T$ is real and upper quasitriangular. Lapack 3.0 will contain Fortran code for calculating and reordering - a Gauss implementation is found at http://www.hhs.se/personal/psoderlind/. Matlab code is found at http://www.iies.su.se/data/home/kleinp/. 
property in Proposition 1 of (Blanchard and Kahn 1980), that is, that the number of predetermined variables (number of rows in $Z_{k \theta}$ ) equals the number of stable roots (number of columns in $Z_{k \theta}$ ). Suppose $Z_{k \theta}^{-1}$ exists, then

$$
\theta_{0}=Z_{k \theta}^{-1} k_{0}
$$

From (1) we have $x_{1 t+1}-\mathrm{E}_{t} x_{1 t+1}=\varepsilon_{t+1}$, and (Backus and Driffill 1986) show that $\rho_{2 t+1}-\mathrm{E}_{t} \rho_{2 t+1}=\mathbf{0}_{n_{2} \times 1}$. Stack these expressions as $k_{t+1}-\mathrm{E}_{t} k_{t+1}$ (recall the definition of $k_{t}$ in (5)), and use $k_{t}=Z_{k \theta} \theta_{t}$ from (10) to express it as

$$
Z_{k \theta}\left(\theta_{t+1}-\mathrm{E}_{t} \theta_{t+1}\right)=\left[\begin{array}{l}
\varepsilon_{t+1} \\
\mathbf{0}_{n_{2} \times 1}
\end{array}\right] .
$$

Invert this expression and substitute for $\mathrm{E}_{t} \theta_{t+1}$ from (9)

$$
\theta_{t+1}=S_{\theta \theta}^{-1} T_{\theta \theta} \theta_{t}+Z_{k \theta}^{-1}\left[\begin{array}{l}
\varepsilon_{t+1} \\
\mathbf{0}_{n_{2} \times 1}
\end{array}\right] \text {. }
$$

which together with (11) summarizes the dynamics of the model. This is can be expressed in term of the original variables by recalling (5) and (10)

$$
\left[\begin{array}{l}
x_{1 t+1} \\
\rho_{2 t+1}
\end{array}\right]=Z_{k \theta} S_{\theta \theta}^{-1} T_{\theta \theta} Z_{k \theta}^{-1}\left[\begin{array}{l}
x_{1 t} \\
\rho_{2 t}
\end{array}\right]+\left[\begin{array}{l}
\varepsilon_{t+1} \\
\mathbf{0}_{n_{2} \times 1}
\end{array}\right],
$$

with $x_{10}$ given and $\rho_{20}=\mathbf{0}_{n_{2} \times 1}$. The same equations also give

$$
\left[\begin{array}{c}
x_{2 t} \\
u_{t} \\
\rho_{1 t}
\end{array}\right]=Z_{\lambda \theta} Z_{k \theta}^{-1}\left[\begin{array}{c}
x_{1 t} \\
\rho_{2 t}
\end{array}\right]
$$

\subsection{An Optimal "Simple Rule"}

The policy maker could alternatively commit to a simple decision rule of the form $u_{t}=-F x_{t}$, as discussed in (Levine and Currie 1987). There may be restrictions on the elements in $F$, and the vector $x_{t}$ could be augmented compared to what is necessary to solve for the unrestricted optimal policy above. 
Substituting for $u_{t}=-F x_{t}$ in (1) gives

$$
\left[\begin{array}{l}
x_{1 t+1} \\
\mathrm{E}_{t} x_{2 t+1}
\end{array}\right]=(A-B F)\left[\begin{array}{l}
x_{1 t} \\
x_{2 t}
\end{array}\right]+\left[\begin{array}{l}
\varepsilon_{t+1} \\
\mathbf{0}_{n_{2} \times 1}
\end{array}\right] .
$$

Taking expectations conditional on the information set in $t$, and letting $k_{t}=x_{1 t}$ and $\lambda_{t}=x_{2 t}$ give the same type of problem as in (5), but with $G=I$ and $D=$ $A-B F$. This means that we can set $S$ and $Q$ in (6) equal to $I$ and $Z$, respectively. This is the original, not generalized, Schur decomposition ? $^{3}$ In contrast to the Jordan decomposition used by (Blanchard and Kahn 1980), it is relatively easy to calculate with good numerical precision (see (Golub and van Loan 1989)), and is frequently available in software libraries.

Provided the $F$ matrix implies a unique equilibrium ( $Z_{k \theta}$ is invertible) the solution is given by the analogies to (14) and (15),

$$
\begin{aligned}
x_{1 t+1} & =M x_{1 t}+\varepsilon_{t+1}, \text { where } M=Z_{k \theta} T_{\theta \theta} Z_{k \theta}^{-1}, \text { and } \\
x_{2 t} & =C x_{1 t}, \text { where } C=Z_{\lambda \theta} Z_{k \theta}^{-1} .
\end{aligned}
$$

It is straightforward to show that the loss function value, for a given $F$ matrix, is

$$
J_{0}=x_{10}^{\prime} V x_{10}+\frac{\beta}{1-\beta} \operatorname{trace}(V \Sigma),
$$

where the matrix $V$ is the fixed point in the iteration ("backwards in time") on

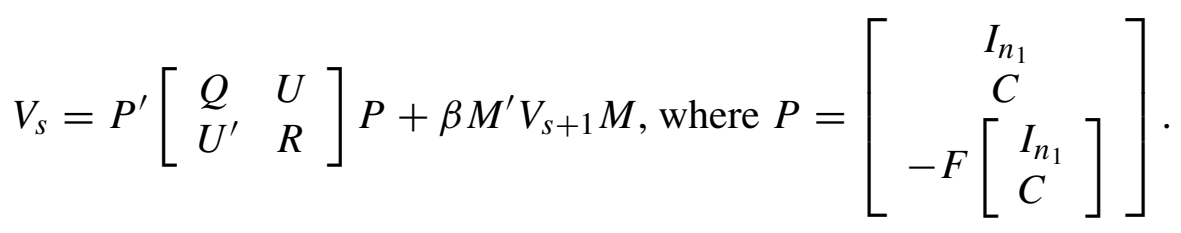

The optimal simple rule minimizes the loss function $(19)$ by choosing the optimal elements in the decision rule $F$, subject to the restrictions that the decision

3 This decomposition is used to solve the eigenvalue problem $D x=\lambda x$. The results in the text hold also for the real Schur decomposition, where $Z$ is a real orthogonal matrix, and $T$ a real upper quasi-triangular matrix. If $A-B F$ has linearly independent eigenvectors, then a spectral decomposition can be used. 
rule should give a unique equilibrium and that $x_{10}$ is given. This rule must typically be found by a non-linear optimization algorithm. It is not certainty equivalent - it depends on the covariance matrix, $\Sigma$, and the initial state vector, $x_{10}$ (see (Currie and Levine 1993)).

\subsection{Optimal Policy under Discretion}

In the discretionary case, the policy maker reoptimizes every period by taking the process by which private agents form their expectations as given - and where the expectations are consistent with actual policy. Since the model is linear-quadratic, the solution in $t+1$ gives a value function which quadratic in the state variables, $x_{1 t+1}^{\prime} V_{t+1} x_{1 t+1}+v_{t+1}$, and a linear relation between the forward looking variables and the state variables, $x_{2 t+1}=C_{t+1} x_{1 t+1}$. Private agents form expectations about $x_{2 t+1}$ accordingly. The value function of the policy maker in $t$ will then satisfy the Bellman equation

$$
\begin{aligned}
x_{1 t}^{\prime} V_{t} x_{1 t}+v_{t} & =\min _{u_{t}}\left[x_{t}^{\prime} Q x_{t}+2 x_{t}^{\prime} U u_{t}+u_{t}^{\prime} R u_{t}+\beta \mathrm{E}_{t}\left(x_{1 t+1}^{\prime} V_{t+1} x_{1 t+1}+v_{t+1}\right)\right] \\
\text { s.t. } \mathrm{E}_{t} x_{2 t+1} & =C_{t+1} \mathrm{E}_{t} x_{1 t+1},(1), \text { and } x_{1 t} \text { given. }
\end{aligned}
$$

Combine the two restrictions to rewrite the problem. Partition the matrices $A$ and $B$ in (1), and $Q$ and $U$ in (21) conformably with $x_{1 t}$ and $x_{2 t}$. Rewrite (21) as

$$
\begin{aligned}
x_{1 t}^{\prime} V_{t} x_{1 t}+v_{t} & =\min _{u_{t}}\left[x_{1 t}^{\prime} Q_{t}^{*} x_{1 t}+2 x_{1 t}^{\prime} U_{t}^{*} u_{t}+u_{t}^{\prime} R_{t}^{*} u_{t}+\beta \mathrm{E}_{t}\left(x_{1 t+1}^{\prime} V_{t+1} x_{1 t+1}+v_{t+1}\right)\right] \\
\text { s.t. } x_{1 t+1} & =A_{t}^{*} x_{1 t}+B_{t}^{*} u_{t}+\varepsilon_{t+1} \text { and } x_{1 t} \text { given, }
\end{aligned}
$$


where the "starred" matrices are defined as

$$
\begin{aligned}
& D_{t}=\left(A_{22}-C_{t+1} A_{12}\right)^{-1}\left(C_{t+1} A_{11}-A_{21}\right) \\
& G_{t}=\left(A_{22}-C_{t+1} A_{12}\right)^{-1}\left(C_{t+1} B_{1}-B_{2}\right) \\
& A_{t}^{*}=A_{11}+A_{12} D_{t} \\
& B_{t}^{*}=B_{1}+A_{12} G_{t} \\
& Q_{t}^{*}=Q_{11}+Q_{12} D_{t}+D_{t}^{\prime} Q_{21}+D_{t}^{\prime} Q_{22} D_{t} \\
& U_{t}^{*}=Q_{12} G_{t}+D_{t}^{\prime} Q_{22} G_{t}+U_{1}+D_{t}^{\prime} U_{2} \\
& R_{t}^{*}=R+G_{t}^{\prime} Q_{22} G_{t}+G_{t}^{\prime} U_{2}+U_{2}^{\prime} G_{t} .
\end{aligned}
$$

(The $D_{t}$ and $G_{t}$ matrices are used to substitute for $x_{2 t}$ according to $x_{2 t}=D_{t} x_{1 t}+$ $\left.G_{t} u_{t} \cdot\right)$

The first order condition of 22) with respect to $u_{t}$ are

$$
u_{t}=-F_{1 t} x_{1 t}, F_{1 t}=\left(R_{t}^{*}+\beta B_{t}^{*^{\prime}} V_{t+1} B_{t}^{*}\right)^{-1}\left(U_{t}^{*^{\prime}}+\beta B_{t}^{*^{\prime}} V_{t+1} A_{t}^{*}\right) .
$$

Combining with (22) gives

$$
\begin{aligned}
x_{2 t} & =C_{t} x_{1 t}, \text { with } C_{t}=D_{t}-G_{t} F_{1 t}, \text { and } \\
V_{t} & =Q_{t}^{*}-U_{t}^{*} F_{1 t}-F_{1 t}^{\prime} U_{t}^{* \prime}+F_{1 t}^{\prime} R_{t}^{*} F_{1 t}+\beta\left(A_{t}^{*}-B_{t}^{*} F_{1 t}\right)^{\prime} V_{t+1}\left(A_{t}^{*}-B_{t}^{*} F_{1 t}\right) .
\end{aligned}
$$

Iterating until convergence ("backwards in time") on (23)-26) is the algorithm used by (Oudiz and Sachs 1985) as well as (Backus and Driffill 1986). It should be started with a symmetric positive definite $V_{t+1}$ and some $C_{t+1}$ (a matrix with zeros seems to work in most cases).

In many cases $F_{1 t}$ and $C_{t}$ converge to constants $F_{1}$ and $C$, even if the iterations may be time consuming $4^{4}$ The first $n_{1}$ equations in $(1)$ can then be written

$$
x_{1 t+1}=\left(A_{11}+A_{12} C-B_{1} F_{1}\right) x_{1 t}+\varepsilon_{t+1},
$$

and the other variables are calculated as $x_{2 t}=C x_{1 t}$ and $u_{t}=-F_{1} x_{1 t}$.

\footnotetext{
4 The general properties of this algorithm are unknown. Practical experience suggests that it is often harder to find the discretionary equilibrium than the commitment equilibrium. It is unclear if this is due to the algorithm.
} 


\section{Maximum Likelihood Estimation of the Model}

In the models discussed in the previous section, the structural parameters are mapped into the evolution of the economy (1) and loss function (2). These give equilibrium laws of motion of the relevant state variables: VAR(1) systems for $x_{1 t}$ in the discretionary case, (27), and in the simple rule, (17), and a VAR(1) for $x_{1 t}$ and $\rho_{2 t}$ in the commitment case, (14). We write this as

$$
\alpha_{t}=\mu+\Phi \alpha_{t-1}+\eta_{t}
$$

We typically have data on a vector $y_{t}$, which is a non-invertible linear combination of the state variables

$$
y_{t}=\lambda+\Gamma \alpha_{t}+\epsilon_{t},
$$

where $\epsilon_{t}$ are some measurement errors. In many cases, $\Gamma$ is known and the measurement errors are zero; in other cases, they need to be estimated. In practice, the measurement errors are often used to translate the model definitions to something corresponding to data and to avoid stochastic singularity (fewer shocks than data series), which makes maximum likelihood estimation very tricky (singular covariance matrix).

The equations (28) and (29) are the starting point for a time-invariant Kalman filter. With normally distributed shocks, it is ideal for building up the likelihood function for sample of $y_{t}$ recursively 5

This likelihood function can be maximized (with a non-linear optimization algorithm) by choosing the structural parameters, which give the matrices $\Phi$ and $\Gamma$ as well as the covariance matrices of the shocks. In the estimation, the filter needs to be initialized with an estimate of the state vector and a covariance matrix of the associated estimation errors. For a model with stationary variables, the

\footnotetext{
5 See, for instance, (Harvey 1989), (Lütkepohl 1993), or (Hamilton 1994).
} 
natural starting point is the uninformed forecast, that is, the unconditional mean and the unconditional covariance matrix. For a given transition matrix $\Phi$ and a covariance matrix of $\eta_{t}$, these are $(I-\Phi)^{-1} \mu$, and the matrix which solves $\Omega=\Phi \Omega \Phi^{\prime}+\operatorname{Cov}\left(\eta_{t}\right)$ (iterate by starting from $\Omega=\mathbf{0}$ ), respectively.

\section{An Example}

To illustrate the properties of this estimation approach, I do a Monte Carlo experiment with the model estimated in (Söderlind 1997). This is a simplified version of the model in (Fuhrer and Moore 1995). The IS curve for detrended log output, $y_{t}$, is

$$
y_{t}=\alpha_{1} y_{t-1}+\alpha_{2} y_{t-2}+\alpha_{r} r_{t-1}+\varepsilon_{y t},
$$

where $\varepsilon_{y t}$ is an output shock. The long ex ante real interest rate, $r_{t}$, obeys an approximate and risk neutral arbitrage condition for a ten year real coupon bond

$$
r_{t}=\frac{1}{41} \sum_{s=0}^{\infty}\left(\frac{40}{41}\right)^{s} \mathrm{E}_{t}\left(i_{t+s}-\pi_{t+1+s}\right),
$$

where $i_{t}$ is the annualized one quarter nominal interest rate (the policy instrument), and $\pi_{t}$ the annualized one quarter inflation rate, $\pi_{t}=4\left(p_{t}-p_{t-1}\right)$. Wage contracts negotiated in $t$ specify a flat nominal wage, $w_{t}$, for three quarters, $t$ to $t+2$. A fraction $\theta_{1} / \theta_{0}$ of these contracts "survive" until in $t+1$, and a fraction $\left(1-\theta_{0}-\theta_{1}\right) / \theta_{0}$ until $t+2$. The log price level is the average of the wage contracts still in effect

$$
p_{t}=\theta_{0} w_{t}+\theta_{1} w_{t-1}+\left(1-\theta_{0}-\theta_{1}\right) w_{t-2} .
$$

Nominal wage contracts are set so the current real contract wage equals the average real contract price index, $v_{t}$, expected to hold over the contract period, ad- 
justed for demand pressure and wage shocks, $\varepsilon_{p t}$,

$$
\begin{aligned}
w_{t} & =p_{t}+\theta_{0}\left(v_{t}+\gamma y_{t}\right)+\theta_{1} \mathrm{E}_{t}\left(v_{t+1}+\gamma y_{t+1}\right)+\left(1-\theta_{0}-\theta_{1}\right) \mathrm{E}_{t}\left(v_{t+2}+\gamma y_{t+2}\right)+\varepsilon_{p t}, \\
v_{t} & =\theta_{0}\left(w_{t}-p_{t}\right)+\theta_{1}\left(w_{t-1}-p_{t-1}\right)+\left(1-\theta_{0}-\theta_{1}\right)\left(w_{t-2}-p_{t-2}\right) .
\end{aligned}
$$

I assume that the Fed sets short interest rates to minimize the loss function

$$
L_{t}=\mathrm{E}_{t} \sum_{t=0}^{\infty} \beta^{t}\left[q_{y} y_{t}^{2}+\left(1-q_{y}\right) \pi_{t}^{2}+q_{i} i_{t}^{2}\right],
$$

and that the Fed can commit to a policy rule. It is then straightforward to rewrite the model on the form (1)-(2), with $x_{1 t}=\left(\varepsilon_{p t}, y_{t}, y_{t-1}, \Delta w_{t-1}, \Delta w_{t-2}, \Delta w_{t-3}\right)$, $x_{2 t}=\left(r_{t}, \Delta w_{t}, \mathrm{E}_{t} \Delta w_{t+1}\right)$, and $u_{t}=i_{t}$.

(Söderlind 1997) estimates the model on a sample of quarterly US data from the mid 1960s to the mid 1990s (123 observations): $y_{t}$ is taken to be log real GNP per capita detrended with a linear trend, $\pi_{t}$ the quarterly changes in the consumer price index for urban workers, and $i_{t}$ is assumed to differ from the three month T-bill rate $\left(i_{1, t}\right)$ by an error term only, $i_{1, t}=i_{t}+\varepsilon_{i t}$.

The Monte Carlo experiment generates 3000 samples of "data" by drawing the initial state vector from its unconditional distribution, and then feeding the dynamics with random numbers for the three shocks. The model is then estimated on each of these samples - using either 123 observations, or a smaller sample of 61 observations (which may be more relevant for many data sets).

The results from the 3000 Monte Carlo simulations are summarized in Table 1. The means of the estimates are generally close to the true values, even if the short sample exaggerates the effectiveness of monetary policy by making output too responsive to real interest rates ( $\alpha_{r}$ too low), and wage setting too responsive to output ( $\gamma$ too high). At the same time, it underestimates the willingness to use monetary policy ( $q_{i}$ too high). These parameters, and also the weight on output in the loss function, $q_{y}$, have considerably higher standard deviations than 
$\underline{\text { Point estimates }}$

$\underline{\text { Standard deviations }}$

\begin{tabular}{lcccccc} 
& \multicolumn{3}{c}{ Point estimates } & \multicolumn{2}{c}{ Standard deviations } \\
& Asymptotic & \multicolumn{2}{c}{ Simulations } & Asymptotic & \multicolumn{2}{c}{ Simulations } \\
& & $T=123$ & $T=61$ & $T=123$ & $T=123$ & $T=61$ \\
\hline$\alpha_{1}$ & 1.39 & 1.38 & 1.38 & 0.07 & 0.07 & $0.08 \sqrt{2}$ \\
$\alpha_{2}$ & -0.50 & -0.50 & -0.49 & 0.06 & 0.07 & $0.07 \sqrt{2}$ \\
$\alpha_{r}$ & -0.55 & -0.58 & -0.63 & 0.13 & 0.17 & $0.22 \sqrt{2}$ \\
$\operatorname{Std}\left(\varepsilon_{y t}\right)$ & 0.84 & 0.84 & 0.84 & 0.05 & 0.06 & $0.06 \sqrt{2}$ \\
$\theta_{0}$ & 0.62 & 0.66 & 0.65 & 0.10 & 0.12 & $0.10 \sqrt{2}$ \\
$\theta_{1}$ & 0.29 & 0.26 & 0.26 & 0.05 & 0.07 & $0.06 \sqrt{2}$ \\
$\gamma \times 100$ & 0.19 & 0.21 & 0.26 & 0.12 & 0.16 & $0.19 \sqrt{2}$ \\
$\operatorname{Std}\left(\varepsilon_{p t}\right)$ & 0.19 & 0.18 & 0.18 & 0.09 & 0.09 & $0.08 \sqrt{2}$ \\
$q_{y}$ & 0.82 & 0.83 & 0.84 & 0.03 & 0.05 & $0.12 \sqrt{2}$ \\
$q_{i}$ & 0.35 & 0.39 & 0.47 & 0.12 & 0.17 & $0.34 \sqrt{2}$ \\
$\operatorname{Std}\left(\varepsilon_{i t}\right)$ & 1.41 & 1.39 & 1.37 & 0.09 & 0.09 & $0.09 \sqrt{2}$ \\
\hline
\end{tabular}

The parameters values used for simulating the model (the asymptotic point estimates) are taken from (Söderlind 1997). The asymptotic standard deviations are calculated from the theoretical information matrix implied by these parameter values. The table also reports the means and the standard deviations of the 3000 estimates on samples with 123 and 61 observations.

Table 1: Results from maximum likelihood estimation, 3000 repetitions

predicted by asymptotic theory. This is particularly clear for the two weights in the loss function ( $q_{y}$ and $q_{i}$ ) in the short sample: the standard deviations are more than three times larger than predicted by asymptotic theory - and twice as large as expected from the results for the longer sample (the factor $\sqrt{2}$ should, in principle, adjust for the sample length).

The correlations of the parameters estimates (not shown) also show some interesting features, which all work in the direction of "stabilizing" the properties of the model. First, $\alpha_{1}$ and $\alpha_{2}$ are strongly negatively correlated (-0.85), which keeps the autocorrelation of output relatively stable across simulations. Second, $a_{r}$ and $q_{i}$ are negatively correlated (-0.65), which keeps the effect of monetary policy on output and inflation relatively constant. Third and finally, the average contract length $\left(\theta_{0}+2 \theta_{1}+3\left(1-\theta_{0}-\theta_{1}\right)\right)$ and $\gamma$ are strongly positively correlated (0.83), which keeps the effect of a price shock on inflation and output relatively 
unchanged across simulations.

\section{References}

Backus, D., and J. Driffill, 1986, The consistency of optimal policy in stochastic rational expectations models, CEPR Discussion Paper No. 124.

Blanchard, O.J., and C.M. Kahn, 1980, The solution of linear difference equations under rational expectations, Econometrica 48, 1305-1311.

Currie, D., and P. Levine, 1993, Rules, Reputation and Macroeconomic Policy Coordination (Cambridge University Press: Cambridge).

Fuhrer, Jeffrey C., and George Moore, 1995, Monetary policy trade-offs and the correlation between nominal interest rates and real output, American Economic Review 85, 219-239.

Golub, Gene H., and Charles F. van Loan, 1989, Matrix Computations (The John Hopkins University Press: Baltimore, MD) 2nd edn.

Hamilton, James D., 1994, Time Series Analysis (Princeton University Press: Princeton).

Harvey, Andrew C., 1989, Forecasting, Structural Time Series Models and the Kalman Filter (Cambridge University Press: Cambridge).

Klein, Paul, 2000, Using the generalized schur form to solve a multivariate linear rational expectations model, Journal of Economic Dynamics and Control 24, $1405-1423$. 
Levine, Paul, and David Currie, 1987, The design of feedback rules in linear stochastic rational expectations models, Journal of Economic Dynamics and Control 11, 1-28.

Lütkepohl, Helmut, 1993, Introduction to Multiple Time Series (Springer-Verlag: Berlin) 2nd edn.

Oudiz, Gilles, and Jeffrey Sachs, 1985, International policy coordination in dynamic macroeconomic models, in Willem H. Buiter, and Richard C. Marston, ed.: International Economic Policy Coordination (Cambridge University Press: Cambridge).

Sims, Christopher A., 2001, Solving linear rational expectations models, Journal of Computational Economics 20, 1-20.

Söderlind, Paul, 1997, Monetary policy and the Fisher effect, Working Paper No. 159, Stockholm School of Economics. 